\title{
Liderazgo transformacional y engagement en directivos en salas de juego de Mar del Plata
}

\section{Analía Soledad Gabriela Caridi ${ }^{1}$, Soledad Beatriz del Otero' ${ }^{2}$, Héctor José Savino ${ }^{3}$, Yamila Fernanda Silva Peralta ${ }^{4}$}

\author{
Facultad de Psicología de la Universidad Nacional de Mar del Plata. ${ }^{1}$ \\ Facultad de Psicología de la Universidad Nacional de Mar del Plata. ${ }^{2}$ \\ Facultad de Psicología de la Universidad Nacional de Mar del Plata. ${ }^{3}$ \\ Facultad de Psicología de la Universidad Nacional de Mar del Plata, CONICET. ${ }^{4}$
}

(Recibido, de 26 Octubre, 2017; Aceptado, de 24 Abril, 2018)

RESUMEN: Se presenta una investigación descriptiva cuya finalidad es conocer el liderazgo transformacional y engagement en directivos de salas de juego de Mar del Plata-Argentina. Para ello se administró el Multifactor Leadership Questionnaire de Avolio y Bass (2004) y el Utrecht Work Engagement Survey de Schaufeli y Bakker (2003) a 20 directivos que voluntariamente y de forma anónima decidieron completar estos cuestionarios. Los resultados muestran que los niveles bajos y muy bajos en las dimensiones transformacionales y los niveles bajos y medios en las dimensiones de engagement caracterizan a más del $50 \%$ de los directivos. Aplicando pruebas no paramétricas no se han encontrado niveles críticos de asociación entre liderazgo transformacional y engagement.

Palabras clave: liderazgo transformacional, engagement, directivos, salas de juego.

\section{Managers’ transformational leadership and engagement in game rooms at Mar del Plata}

ABSTRACT: A descriptive research is presented which aim is to know about managers' transformational leadership and engagement in game rooms at Mar del Plata-Argentina. For that reason the Multifactor Leadership Questionnaire from Avolio and Bass (2004) and the Utrecht Work Engagement Survey from Schaufeli and Bakker (2003) were administered to 20 managers who completed these surveys in a voluntary and anonymous way. Results show low and very low transformational levels and low and medium engagement levels in more than the $50 \%$ of managers. Non parametric tests were applied and no critical levels were found between transformational leadership and engagement.

Keywords: transformational leadership, engagement, managers, game rooms

Correspondencia: ${ }^{1}$ analiacaridi@hotmail.com.

$$
\begin{aligned}
& 2 \text { soletandil@hotmail.com. } \\
& 3 \text { cabosavino@hotmail.com. } \\
& 4 \text { yamilasilvaperalta@gmail.com. }
\end{aligned}
$$




\section{Introducción}

El presente estudio de carácter descriptivo tiene como finalidad analizar dos variables críticas a la hora de gestionar procesos y resultados organizacionales: el liderazgo transformacional y engagement en directivos de salas de juego de la ciudad de Mar del PlataArgentina. Se plantean como objetivos particulares de esta investigación: describir los niveles transformacionales y de engagement del personal directivo y analizar asociaciones entre los mismos con miras a detectar si existen o no diferencias significativas.

A partir de un recorrido y análisis bibliográfico, se ha pesquisado la incidencia del estilo de liderazgo transformacional en la salud mental de los trabajadores, así como también en la promoción del engagement, el cual se considera uno de los factores protectores contra niveles altos de estrés. A su vez, se advierte la reducida la investigación sobre estas temáticas en trabajadores de salas de juego/casinos, razón por la cual se aspira a realizar un aporte inicial que pudiera servir para posteriores estudios y para el diseño de propuestas concretas de gestión de personal en el marco de este tipo de organizaciones.

\section{Antecedentes teóricos}

Dinh, Lord, Gardner, Meuser, Liden y Hu (2014) desarrollaron un estudio documental con 752 investigaciones publicadas entre los años 2000-2012, considerando como fuentes las siguientes revistas: Leadership Quarterly, Administrative Science Quarterly, American Psychologist, Journal of Management, Academy of Management Journal, Academy of Management Review, Journal of Applied Psychology, Organizational Behavior \& Human Decision Processes, Organizational Science y Personnel Psychology. Los resultados muestran que el 39\% ( $\mathrm{n}=294)$ de las investigaciones se concentran en los modelos denominados neocarismáticos (liderazgos transformacional, carismático, transaccional, ideológico/pragmático, excepcional, abnegado, inspiracional y el efecto Pigmalión). Según Stentz, Plano Clark y Matkin (2012) el estudio del liderazgo tiene una larga trayectoria de tipo cuantitativa. Las investigaciones de los últimos cinco años revisadas a continuación apoyan dicha afirmación.

Mendoza Martínez, Escobar Álvarez y García Rivera (2012) desarrollaron un estudio en una escuela pública de México, los autores subrayan que existen correlaciones directas significativas entre el liderazgo transformacional, el liderazgo transaccional y las variables de resultado; por otra parte, se advierten correlaciones inversas significativas entre el liderazgo transformacional, el liderazgo transaccional y las variables de resultado con el no liderazgo. Zárate y Matviuk, (2012) midieron la relación entre liderazgo e inteligencia emocional en más de 300 empleados de Bogotá, Colombia, para ello administraron el inventario de Kouzes y Posner y el instrumento de Wong y Law de inteligencia emocional, los resultados muestran que quienes puntúan alto en inteligencia emocional lideran más eficientemente sus grupos de trabajo. Robles, Contreras, Barbosa y Juárez (2013) también aplicaron el Inventario de Kouzes y Posner en directivos colombianos y mexicanos no encontrando diferencias relevantes entre países. Trógolo, Pereyra y Spoton (2013) coinciden en que el liderazgo transformacional aumenta el bienestar de los trabajadores en una organización saludable; según sus resultados el liderazgo transformacional influye positivamente en el engagement y negativamente en el 
burnout. Arenas Ortiz y Andrade Jaramillo (2013) estimaron factores de riesgo psicosocial intralaboral y extralaboral en una industria alimenticia encontrando que un liderazgo inapropiado genera tensión en el trabajo y dificultades en el desarrollo de los trabajadores. En México, Cetina, Ortega y Aguilar (2014) aplicaron la Escala de Habilidades Directivas a 171 trabajadores quienes evaluaron a su superior directo, los resultados muestran que los jefes que evidencian habilidades de relaciones humanas y promueven la visión compartida son más respetados y más favorablemente puntuados en su desempeño. En el mismo año, Páez, Rincón, Astudillo y Bohórquez (2014) analizaron el liderazgo transformacional en directivos del sector floricultor colombiano, las dimensiones transformacionales que despuntan en esta última investigación son influencia idealizada y motivación inspiracional. Collins, Burrus y Meyer (2014) investigaron el impacto de los estilos de liderazgo y género en el arraigo de los subordinados y su satisfacción en el trabajo. El líder orientado hacia el afecto y la lealtad influyen positivamente en el arraigo de las mujeres a cargo, no así en el caso de los varones. Durán Gamba y Castañeda (2015) hicieron un estudio con un diseño multivariable con análisis regresional para describir la relación entre el liderazgo transformacional-transaccional con la conducta de compartir conocimiento de los colaboradores, las correlaciones son positivas para el liderazgo transaccional y negativas para el transformacional. Silva Peralta, Gandoy, Jara y Pacenza (2015) analizaron niveles de empoderamiento de estudiantes y liderazgo transformacional de docentes tutores en el marco de prácticas universitarias curricularizadas. Los resultados indican que un alto nivel de empoderamiento en los estudiantes estaría relacionado con altos niveles en las dimensiones del liderazgo laissez-faire y transaccionales de los docentes-tutores y con la dimensión transformacional estimulación intelectual, las restantes dimensiones transformacionales recibieron bajas puntuaciones.

En relación al engagement, Giraldo y Pico (2012) dirán que se trata de un estado afectivo por el cual las personas se vinculan emocionalmente con su trabajo y lo asocian al bienestar laboral, cuando el empleado está vinculado emocional y conductualmente a la empresa favorece a la productividad de la misma y mejora la relación con los clientes. Luzuriaga, Ubilla y Fierro Ulloa (2015) manifiestan que el engagement es uno de los factores clave al buscar resultados empresariales positivos. Montoya Zuluaga y Moreno (2012) plantean que, frente a situaciones laborales estresantes, si las estrategias de afrontamiento presentan alto nivel de autoeficacia y se focalizan en el problema, hay control emocional y un estado mental engagement, entonces las posibilidades de desarrollar síndrome de burnout disminuyen.

Hasta el momento no se han encontrado investigaciones previas que aborden el estudio de las variables que aquí se proponen en población de trabajadores de salas de juego/casinos, considerando la especificidad de estos trabajos y los altos niveles de estrés que se registran.

\section{Método}

La presente es una investigación descriptiva porque se propone analizar niveles de liderazgo transformacional y de engagement en trabajadores de salas de juego en la ciudad de Mar del Plata.

El liderazgo transformacional se compone según Avolio y Bass (2004) de cinco habilidades: (1) Consideración Individual, se refiere a las capacidades del líder para tratar con cuidado y preocupación al seguidor, de forma tal que se sienta valorado por su aporte y de esta 
manera desarrolle su máximo potencial; (2) Estimulación Intelectual, se refiere a líderes que desafían la forma en que sus seguidores resuelven problemas para que revean y re examinen suposiciones subyacentes a los mismos; (3) Motivación Inspiracional, expresa el comportamiento del líder cuando provee una visión que estimula la energía para lograr altos niveles de desempeño y desarrollo, así como también provee a sus seguidores de trabajo significativo y desafiante; (4) Influencia Idealizada como Atributo, es cuando el líder es visto como un modelo por sus seguidores, los líderes tienen un carácter carismático y apoyan e inculcan orgullo en sus seguidores; y (5) Influencia Idealizada como Comportamiento, refiere a un líder que se comporta de manera ética, moral y consistente, transmitiendo valores congruentes con sus acciones y promoviendo la importancia de una misión colectiva.

El engagement es un estado psicológico positivo relacionado con el trabajo y que está caracterizado por: (1) Absorción o concentración en el trabajo, alude a estar muy involucrado en el trabajo al punto de sentir insatisfacción al dejarlo; (2) Dedicación, se caracteriza por altos niveles de entusiasmo y compromiso con la tarea realizada; y (3) Vigor, se refiere al esfuerzo y persistencia ante las dificultades planteadas por el trabajo (Salanova \& Shaufeli, 2004).

Esta investigación trabajó con la totalidad de directivos de salas de juego de la organización. Se trata de 20 directivos que prestaron consentimiento para participar del estudio. Respecto a los datos sociodemográficos, se trata de directivos comprendidos entre las edades 55 y 65 años, todos masculinos, con una antigüedad en la organización que supera los 30 años y una antigüedad en el cargo directivo de 1 a 4 años de antigüedad.

Se administraron el Multifactor Leadership Questionnaire (MLQ-5x) de Avolio y Bass (2004) que mide los niveles transformacionales y el Cuestionario UWES-17 (Utrecht Work Engagement Survey) de Schaufeli y Bakker (2003) para medir niveles de engagement.

Para acceder a los datos primeramente se solicitó una reunión con el personal y directivos de la oficina de RRHH de la Sala de Juegos, a fin de gestionar la pertinente autorización para la administración de cuestionarios. Tras la reunión se estableció que durante la semana, y en los horarios de descanso del personal, se entregarían los cuestionarios a todos aquellos que se encontraran presentes y que accedieran voluntariamente a participar de la experiencia. Una vez en la sala de descanso, se explicó el motivo de la investigación y la razón que ha llevado a la elección del tema. Se le entregó a cada uno de los voluntarios una copia en blanco del MLQ-5x y el UWES-17 en forma conjunta. Posteriormente, se les informó que, en un lugar visible de la sala, se encontraría depositada una caja-urna para que depositaran los cuestionarios una vez finalizados como una manera de garantizar el anonimato de los participantes.

A continuación, se sistematizaron los datos y se analizaron descriptivos básicos, tablas de contingencia y estadísticos no paramétricos para explorar niveles críticos entre las dimensiones previamente señaladas.

\section{Resultados}

Se exponen a continuación los resultados obtenidos a través de las técnicas administradas a los directivos de salas de juego.

Conforme a los objetivos planteados, se procede a describir en primer lugar el liderazgo transformacional del personal directivo (ver Tabla 1). En la dimensión Consideración 
Individual se evidencian 3 casos en el nivel muy bajo (15\%), 7 casos en el nivel bajo (35\%), 4 casos $(20 \%)$ en el nivel medio, 6 casos $(30 \%)$ en el nivel alto y ningún caso en el nivel muy alto. En la dimensión Estimulación Intelectual se evidencian 5 casos (25\%) en el nivel muy bajo, 7 casos (35\%) en el nivel bajo, 2 casos (10\%) en el nivel medio, 6 casos (30\%) en el nivel alto y ningún caso en el nivel muy alto. En la dimensión Motivación Inspiracional se hallan 4 casos $(20 \%)$ en el nivel muy bajo, 7 casos $(35 \%)$ en el nivel bajo, 7 casos $(35 \%)$ en el nivel medio, ningún caso en el nivel alto y 2 casos (10\%) en el nivel muy alto. En la dimensión Influencia Idealizada como Atributo, a diferencia de las dimensiones anteriores, se hallan 11 casos $(55 \%)$ en el nivel muy bajo, 7 casos $(35 \%)$ en el nivel bajo, ningún caso en el nivel medio, 1 caso (5\%) en el nivel alto y 1 caso (5\%) en el nivel muy alto, por lo tanto, el $90 \%$ de los casos se ubican en el rango muy bajo y bajo. Finalmente, en la dimensión Influencia Idealizada como Comportamiento se hallan 4 casos (20\%) en el nivel muy bajo, 8 casos (40\%) en el nivel bajo, 7 casos (35\%) en el nivel medio, ningún caso en el nivel alto y 1 caso (5\%) en el nivel muy alto.

Tabla1. Distribución de frecuencias y porcentajes para cada dimensión de liderazgo transformacional.

\begin{tabular}{lccc}
\hline Dimensión & Niveles & $F$ & $\%$ \\
\hline Consideración Individual & Muy bajo & 3 & 15,00 \\
& Bajo & 7 & 35,00 \\
& Medio & 4 & 20,00 \\
\hline Estimulación Intelectual & Alto & 6 & 30,00 \\
& Muy bajo & 5 & 25,00 \\
& Bajo & 7 & 35,00 \\
& Medio & 2 & 10,00 \\
Motivación Inspiracional & Alto & 6 & 30,00 \\
& Muy bajo & 4 & 20,00 \\
& Bajo & 7 & 35,00 \\
& Medio & 7 & 35,00 \\
& Muy alto & 2 & 10,00 \\
\hline Influencia Idealizada (Atributo) & Muy bajo & 11 & 55,00 \\
& Bajo & 7 & 35,00 \\
& Alto & 1 & 5,00 \\
& Muy alto & 1 & 5,00 \\
\hline
\end{tabular}

Nota. No se presentan en la tabla los niveles con valor 0 . 
Respecto al nivel de engagement del personal directivo, segundo objetivo de esta investigación (ver Tabla 2), en cuanto al nivel de Absorción no se encontraron casos en el nivel muy bajo, alto y muy alto, son 6 los casos con nivel bajo (30\%) y 14 casos con nivel medio (70\%). Respecto al nivel de Dedicación son 2 casos en nivel muy bajo (10\%) y 4 en nivel bajo (20\%), en el nivel medio se distribuyen 13 casos (65\%) y sólo 1 caso (5\%) lo hace en el nivel alto, no habiendo ningún caso en el nivel muy alto. En la dimensión Vigor los valores obtenidos muestran una mayor dispersión, en los niveles bajos se hallaron 3 casos $(15 \%)$ y 13 casos $(65 \%)$ en los niveles medios, se evidencia 1 caso (5\%) en nivel muy bajo, 2 casos $(10 \%)$ en el nivel alto y 1 caso $(5 \%)$ en el nivel muy alto. Por último, en cuanto al nivel de engagement (puntaje total) y, acompañando los resultados antedichos, se puede apreciar la mayor concentración de valores en los niveles medios con 14 casos $(70 \%)$ y en niveles extremos muy bajo y muy alto ningún caso.

Tabla 2. Distribución de frecuencias y porcentajes para cada dimensión de engagement.

\begin{tabular}{lccc}
\hline Dimensión & Niveles & F & $\%$ \\
\hline Absorción & Bajo & 6 & 30,00 \\
& Medio & 14 & 70,00 \\
\hline Dedicación & Muy bajo & 2 & 10,00 \\
& Bajo & 4 & 20,00 \\
& Medio & 13 & 65,00 \\
& Alto & 1 & 5,00 \\
\hline Vigor & Muy bajo & 1 & 5,00 \\
& Bajo & 3 & 15,00 \\
& Medio & 13 & 65,00 \\
& Alto & 2 & 10,00 \\
& Muy alto & 1 & 5,00 \\
\hline Engagement (puntaje total) & Bajo & 5 & 25,00 \\
& Medio & 14 & 70,00 \\
& Alto & 1 & 5,00 \\
\hline
\end{tabular}

Nota. No se presentan en la tabla los niveles con valor 0 .

Se analizan a continuación asociaciones entre niveles de liderazgo transformacional y niveles de engagement en directivos de salas de juego.

Respecto a la asociación entre niveles de engagement y la dimensión Consideración Individual (CI) (ver Figura 1), los resultados muestran que el 50\% de los directivos se distribuyen en niveles bajos y muy bajos de CI, combinándose sobre todo con niveles medios de engagement. 
Figura 1. Asociación entre nivel de engagement y Consideración Individual (CI).

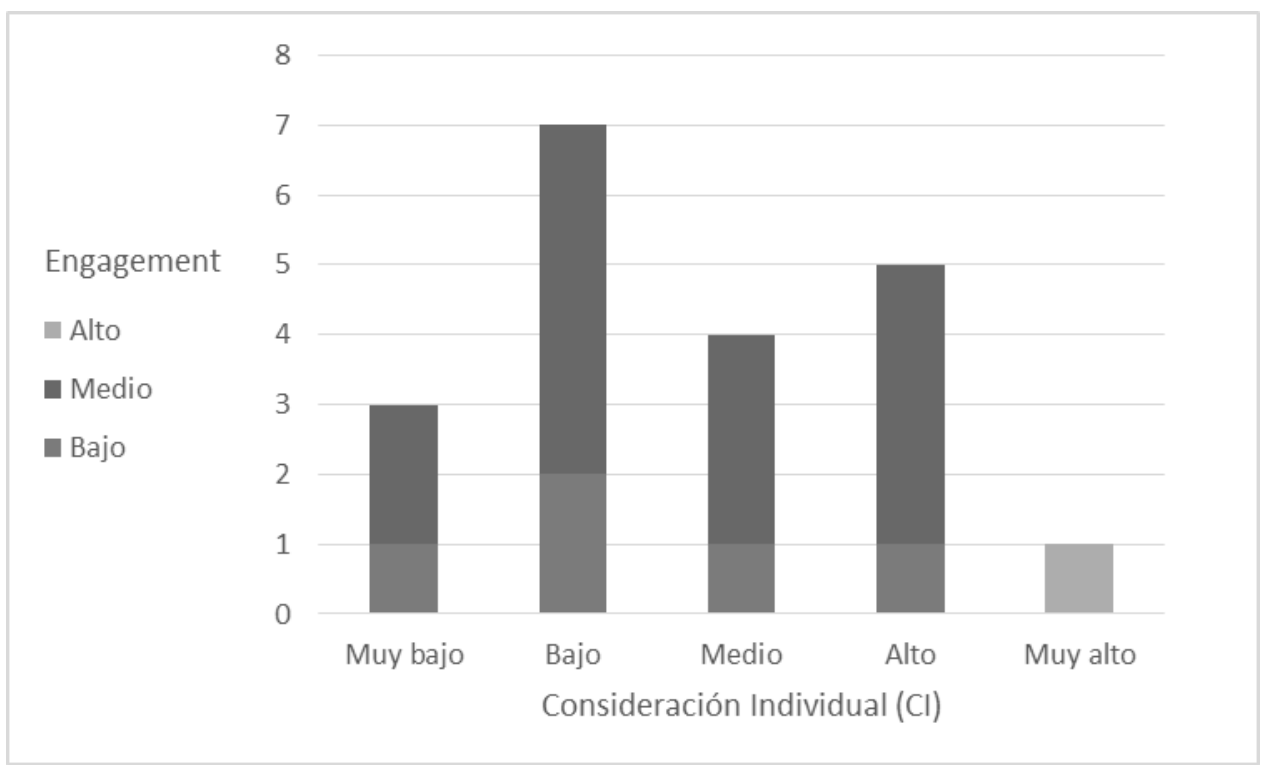

En cuanto a la asociación entre niveles de Engagement y Estimulación Intelectual (EI) (ver Figura 2) se puede apreciar que, de los 20 directivos, 15 se concentran en niveles bajos y muy bajos de EI. Como en la dimensión anterior, se combinan sobre todo con niveles medios de engagement.

Figura 2. Asociación entre nivel de engagement y Estimulación Intelectual (EI).

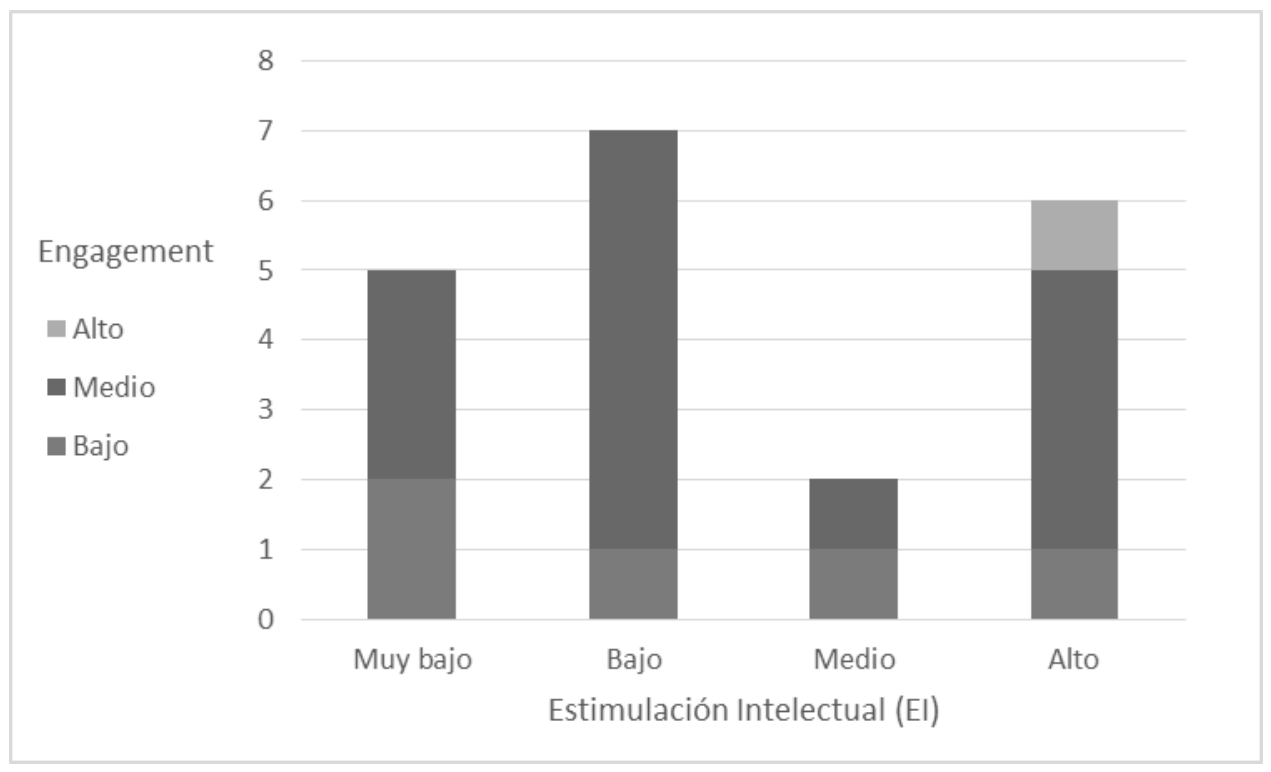

En cuanto a la asociación entre niveles de engagement y Motivación Inspiracional (MI) (ver Figura 3), se distribuyen equitativamente 7 directivos en niveles bajos y 7 en niveles medios combinados en ambos casos con niveles medio de engagement $(\mathrm{n}=10)$ y niveles bajo en menor medida $(\mathrm{n}=4)$. 
Figura 3. Asociación entre nivel de engagement y Motivación Inspiracional (MI).

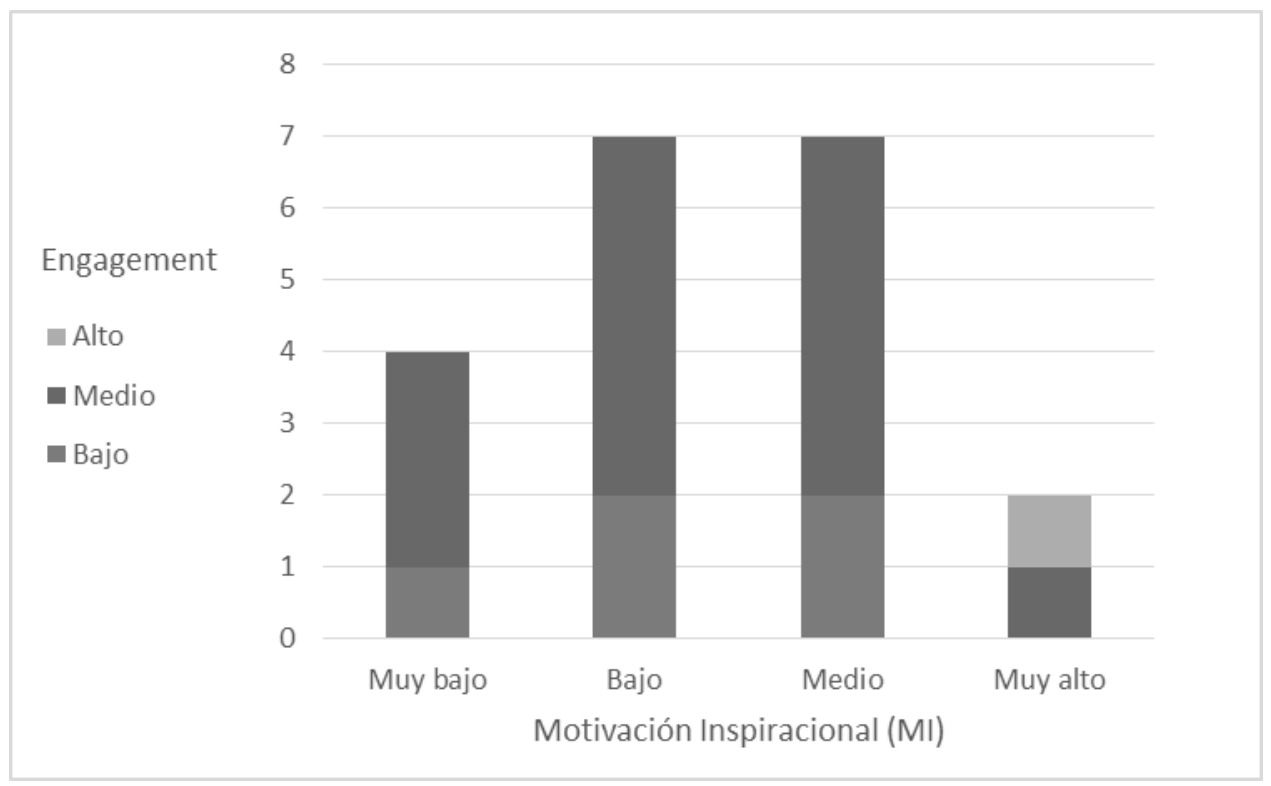

En la asociación entre niveles de engagement e Influencia Idealizada como Comportamiento (IIC) (ver Figura 4) se evidencia que, de los 20 directivos, 8 presentan un nivel bajo de IIC y 7 niveles medios en esta dimensión, en ambos casos están combinados sobre todo con niveles medios de engagement.

Figura 4. Asociación entre nivel de engagement e Influencia Idealizada Comportamiento (IIC).

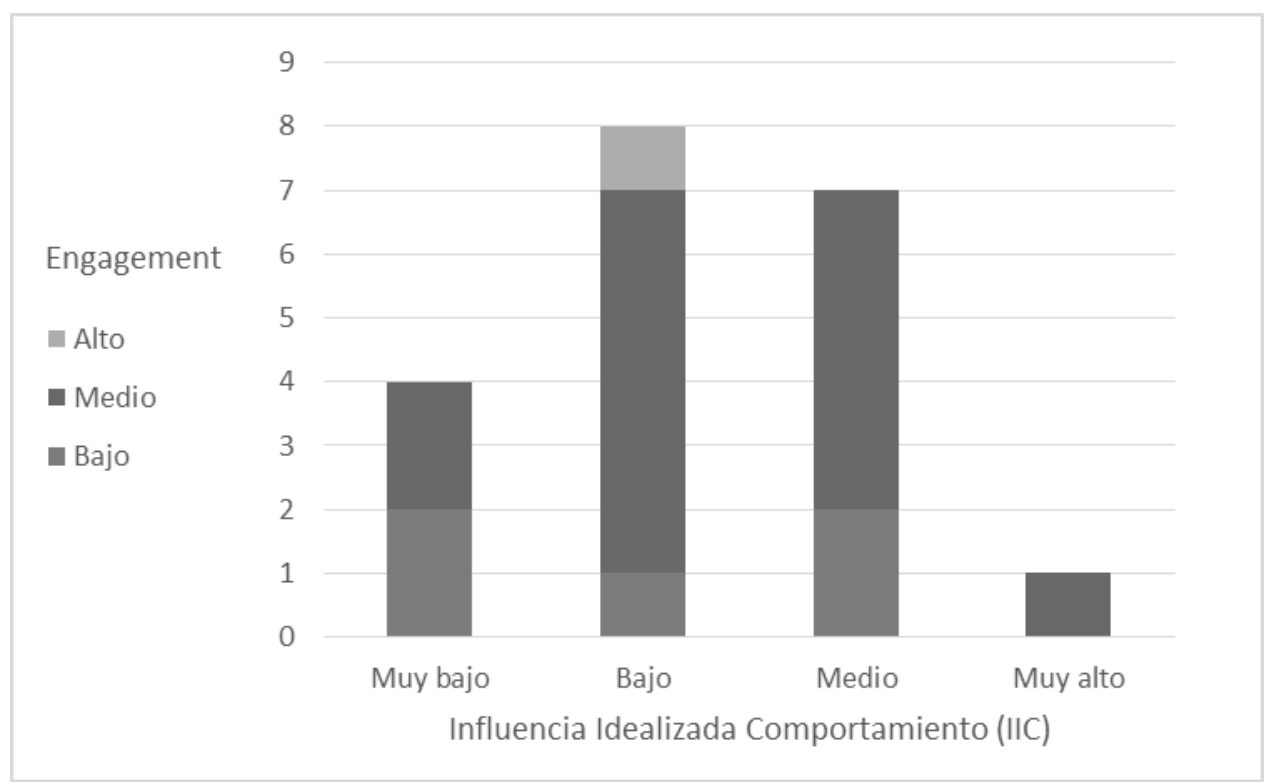

En cuanto a la asociación entre niveles de engagement e Influencia Idealizada como Atributo (IIA) (ver Figura 5), los directivos se distribuyen mayoritariamente en niveles muy bajos y bajos y se asocian en primera instancia con niveles medios de engagement y luego con niveles bajos. 
Figura 5. Asociación entre nivel de engagement e Influencia Idealizada Atributo (IIA).

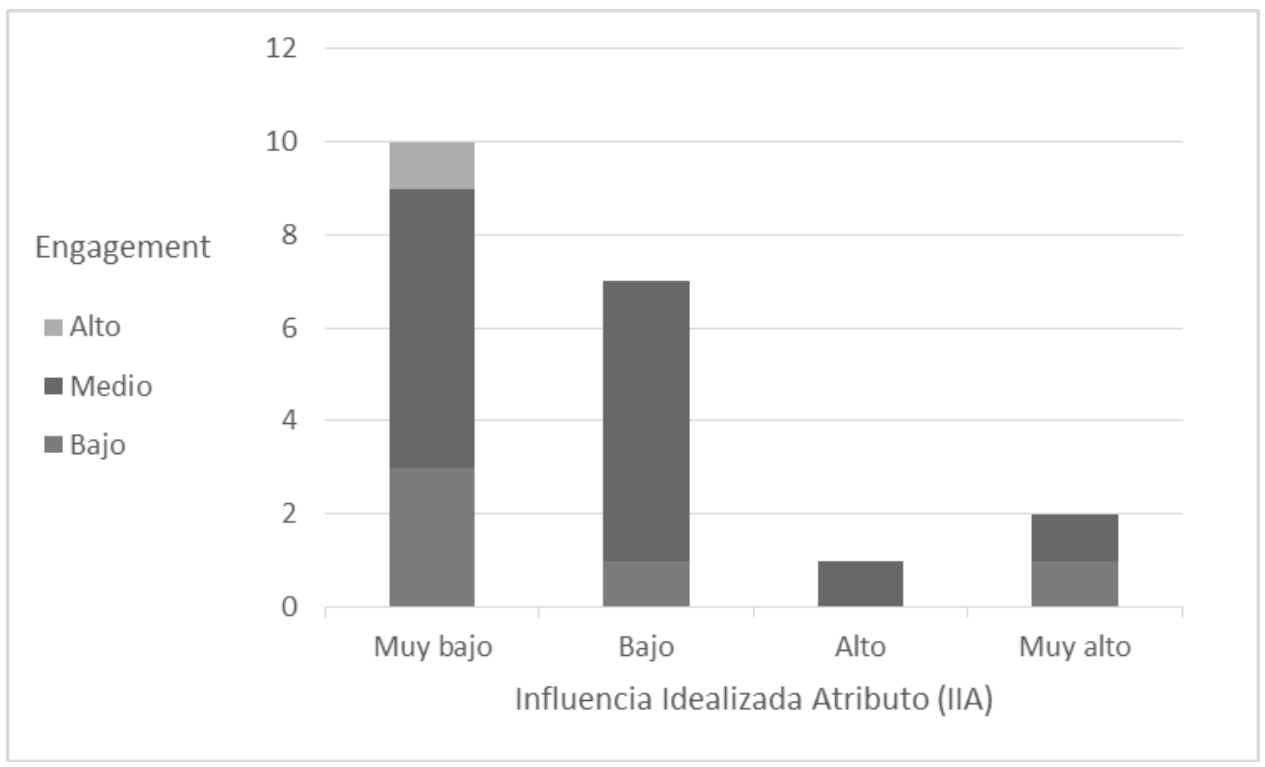

Por último, se presentan los resultados de aplicar estadísticos de contraste no paramétricos utilizando la prueba U de Mann-Whitney. Se realizaron comparaciones dos a dos controlando la significación y tomando como variable de agrupación los niveles medios $(n=14)$ $\mathrm{y}$ bajos $(\mathrm{n}=5)$ de engagement (niveles que acumularon juntos a 19 de los 20 directivos analizados), todo ello para conocer si las dimensiones transformacionales difieren en ambos grupos y si dichas diferencias son significativas. No se hallaron niveles críticos para ninguna de las dimensiones transformacionales (ver Tabla 3).

Tabla 3. Niveles transformacionales según nivel de engagement medio/ alto.

\begin{tabular}{lccccc}
\hline & CI & EI & MI & IIA & IIC \\
\hline U de Mann-Whitney & 30,500 & 30,500 & 33,500 & 28,000 & 21,000 \\
W de Wilcoxon & 45,500 & 45,500 & 48,500 & 43,000 & 36,000 \\
Z &,- 434 &,- 436 &,- 147 &,- 686 & $-1,444$ \\
Sig. asintót. (bilateral) &, 664 &, 663 &, 883 &, 493 &, 149 \\
Sig. exacta [2*(Sig. unilateral)] &, 687 &, 687 &, 893 &, 559 &, 219 \\
\hline
\end{tabular}

Nota. CI es Consideración Individual, EI es Estimulación Intelectual, MI es Motivación Inspiracional, II (A) es Influencia Idealizada (Atributo), II (C) es Influencia Idealizada (Comportamiento).

\section{Discusión y conclusiones}

En la presente investigación se analizaron el liderazgo transformacional y el engagement en 20 directivos de salas de juego de la ciudad de Mar del Plata. Los puntajes obtenidos en las dimensiones transformacionales de los directivos son bajos y muy bajos en porcentajes cercanos al 50\%, a excepción de la dimensión Consideración Individual que tiene dispersión en diferentes niveles y la dimensión Influencia Idealizada como Atributo que acumula el 90\% de los casos en los niveles muy bajo y bajo. 
Tal como se ha anticipado al inicio del presente estudio, se estima interesante explorar la asociación entre los estilos de liderazgo y el engagement en virtud de la repercusión que el primero tiene en la salud mental de los trabajadores y en propiciar un clima de trabajo favorable. En esta línea, se coincide con los aportes de diversos autores que han puesto de manifiesto la incidencia del estilo de liderazgo en la promoción de organizaciones saludables, tal es el caso de Moreno y Velásquez (2011) quienes sugieren que una organización debe preocuparse por aumentar el engagement considerando el talento humano y disminuir el burnout y enfatizan la importancia de que los líderes transformacionales actúen como referentes y puedan establecer el engagement como cultura de la organización. Otros autores que coinciden en que el liderazgo transformacional aumenta el bienestar de los trabajadores en una organización saludable son Trógolo, Pereyra y Spoton (2013), ellos sostienen que las organizaciones saludables potencian habilidades directivas orientadas a la estimulación intelectual, teniendo en cuenta las aspiraciones y necesidades individuales de los trabajadores. Mendoza Martínez, Escobar Álvarez y García Rivera (2012), quienes subrayan que el estilo de liderazgo transformacional se enfoca en elevar el interés de los seguidores, en la promoción de la autoconciencia y en la asunción de la misión organizacional, superando los límites de los auto-intereses en pos del interés por el bienestar del grupo. De Vega, López y Silva Peralta (2012) expresan que en la actualidad las organizaciones requieren líderes genuinos con habilidades sociales que se orienten al cambio, que puedan promover compromiso de sus seguidores en este proceso transformador. Los directivos engaged tienden a crear un entorno de trabajo más desafiante y buscan más recursos para resolverlo, de este modo mantienen su compromiso porque pueden transformar su trabajo y presentan la autonomía para enfrentarse a nuevos desafíos.

Estos antecedentes permiten reflexionar acerca de posibles asociaciones entre los niveles bajos y medios en las dimensiones de engagement y los bajos niveles transformacionales en los directivos analizados en la presente investigación. Aplicando estadísticos no paramétricos no se observaron diferencias significativas respecto a la distribución de las dimensiones transformacionales según sean los niveles de engagement. Los resultados de la presente investigación evidencian un perfil de directivo con reducida capacidad para influir en los seguidores inspirándoles orgullo y respeto, con dificultad para transmitir valores congruentes con sus acciones y promover la importancia de una misión colectiva y una visión de futuro clara, deseable y significativa, que permitiera persuadir y desafiar con entusiasmo y optimismo a los seguidores y despertar el espíritu de equipo, así como con dificultad para potenciar la creatividad e innovación en los seguidores y tratar a cada uno de ellos de manera individual, única y particular. Los resultados obtenidos es poner en evidencia un contexto laboral que resulta tóxico y no promueve el desarrollo de habilidades transformacionales y la promoción de trabajadores engaged. Se requiere continuar investigando este contexto laboral particular con miras a identificar aquellos factores que facilitarían ambientes de trabajo saludables y, de esta forma, prevenir patologías psicolaborales.

\section{Limitaciones y futuras líneas de investigación}

Si bien se considera que el presente estudio resulta ser un aporte útil en una temática poco investigada en trabajadores de salas de juego a nivel local, el mismo presenta como limitación que sólo se ha estimado la autopercepción del liderazgo del personal directivo, no 
habiéndose indagado acerca de la percepción que el personal operativo tiene de sus líderes. Esta decisión metodológica se apoyó en la garantía de confidencialidad del dato, si los directivos se auto-evaluaban y eran evaluados por sus seguidores, se perdía el anonimato del directivo. Respecto a esta cuestión, Cruz Ortiz y Salanova (2011) realizan un aporte interesante a partir de un análisis descriptivo y correlacional que llevan a cabo con el objetivo de evaluar la existencia o no de percepciones compartidas entre equipos de trabajo y supervisores, en cuanto al liderazgo transformacional. Los resultados de su estudio arrojan que existe un gran desajuste en relación a lo percibido por el supervisor y sus equipos de trabajo, siendo que la percepción que los supervisores tienen sobre sí mismos sobre su forma de liderazgo es más elevada en relación a las percepciones que manifiestan sus equipos de trabajo. Los autores sugieren que esta discordancia podría afectar la eficacia colectiva, el bienestar y rendimiento de los equipos de trabajo, por lo que podría resultar de interés indagar sobre esta temática en futuras investigaciones. Otro tema a considerar en futuras investigaciones es la comparativa entre directivos según sean los años de antigüedad en el puesto de dirección, ya que la trayectoria en el puesto también influiría en la posibilidad de desarrollar ciertas competencias de liderazgo.

No se han encontrado niveles críticos de asociación entre niveles de engagement y de liderazgo transformacional en la muestra analizada, futuras investigaciones tendrían que ampliar el número de sujetos de la muestra para contrastar con estos resultados, así como definir estudios que contemplen diversas organizaciones y niveles jerárquicos y no jerárquicos intra e inter-organizacionales.

Por fin, futuras investigaciones deberían abordar estas temáticas con metodologías de corte cualitativo para aportar contenidos estratégicos a la hora de pensar en el diseño de organizaciones transformadoras y saludables que promuevan el engagement de sus trabajadores.

\section{Referencias}

Arenas Ortiz, F. \& Andrade Jaramillo, V. (2013). Factores de riesgo psicosocial y compromiso (engagement) con el trabajo en una organización del sector salud de la ciudad de Cali. Pensamiento psicológico, 11(1), 99-113.

Avolio, B. J., \& Bass, B. M. (2004). Multifactor leadership questionnaire. Third Edition Manual and sampler set. California: Mind Garden, Inc.

Cetina, T., Ortega, I. \& Aguilar, C. (2010). Habilidades directivas desde la percepción de los subordinados: Un enfoque relacional para el estudio del liderazgo. Psicoperspectivas, 9(1), 124-137.

Collins, B. J., Burrus, C., J. \& Meyer, R., D (2014). Gender differences in the impact of leadership styles on subordinate embeddedness and job satisfaction. The Leadership Quarterly, 25(4), 660-671.

De Vega, R., López, A., \& Silva Peralta, Y. (2012). Habilidades transformacionales en el liderazgo de coordinadores y voluntarios. Ponencia presentada en IV Jornadas de Administración del NEA. II Encuentro Internacional de Administración de la Región Jesuítico-Guaraní. Posadas, Misiones.

Dinh, J. E., Lord, R. G., Gardner, W. L. , Meuser, J. D., Liden, R. C. A. \& Hu, J., (2014). Leadership theory and research in the new millennium: Current theoretical trends and changing perspectives. The Leadership Quarterly, 25, 36-62. 
Durán Gamba, M.G., \& Castañeda, D.I. (2015). Relación entre liderazgo transformacional y transaccional con la conducta de compartir conocimiento en dos empresas de servicios. Acta Colombiana de Psicología, 18(1), 135-147.

Giraldo, V., \& Pico, M. (2012). Engagement vínculo emocional del empleado con la organización. Recuperado el 10 de octubre de http://intellectum.unisabana.edu.co/bitstream/handle/10818/3957/MARTHA\%20JULISS A $\% 20$ PICO $\% 20$ FINAL.pdf?sequence $=3$

Luzuriaga Ubilla, D. L., \& Fierro Ulloa, I. (2015). Análisis del talento humano en el desarrollo de las empresas: engagement. Revista Res non verba, 0(7), 91-105.

Mendoza Martínez, I., Escobar Álvarez, G., \& García Rivera, B. (2012). Influencia del liderazgo transformacional en algunas variables de satisfacción organizacional en personal docente y administrativo de una institución pública de educación media superior. Revista del Centro de Investigación. Universidad La Salle, 38(10), 189-206.

Montoya Zuluaga, P. A., \& Moreno, S. (2012). Relación entre síndrome de burnout, estrategias de afrontamiento y engagement. Psicología desde el Caribe, 29(1), 205-227.

Moreno, A. M., \& Velásquez, A. M. (2011). El engagement: un constructo que promueve la calidad de vida en las organizaciones. Recuperado el 10 de octubre de 2017 de http://intellectum.unisabana.edu.co/bitstream/handle/10818/3666/Ana\%20Mar\%C3\%ADa \%20Moreno\%20Rodr\%C3\%ADguez_\%20Trabajo.pdf?sequence=1\&isAllowed=y\%20)

Páez, I, Rincón, A., Astudillo, M. \& Bohórquez, S (2014). Un estudio de casos sobre liderazgo transformacional y competencias directivas en el sector floricultor de Colombia. Revista Escuela de Administración de Negocios, 76, 22-43.

Robles, V. H., Contreras, F., Barbosa, D. \& Juárez, F. (2013). Liderazgo en directivos colombianos vs. mexicanos. Un estudio comparativo. Investigación \& Desarrollo, 21(2), 395-418.

Salanova, M., \& Shaufeli, W. (2004). El engagement de los empleados: un reto emergente para la dirección de los recursos humanos. Revista Estudios Financieros, 62(216), 109-138.

Schaufeli, W., \& Bakker, A. (2003). UWES Utrecht Work Engagement Scale. Escala Utrecht de Engagement en el Trabajo. Preliminary Manual. Ultrescht: Occupational Health Psychology Unit, Utrecht University.

Silva Peralta, Y., Gandoy, F., Jara, C., Pacenza, M. I. (2015). Liderazgo del docente y niveles de empoderamiento de los estudiantes en un seminario de prácticas comunitarias de una universidad pública argentina. Cuadernos de Administración, 31(54), 68-79.

Stentz, J., E., Plano Clark, V. L. \& Matkin, G. S (2012). Applying mixed methods to leadership research: A review of current practices. The Leadership Quarterly, 23, 1173-1183.

Trógolo, M., Pereyra, A., \& Spoton, C. (2013). Impacto de diferentes estilos de liderazgo sobre el engagement y burnout. Evidencia en una muestra de trabajadores argentinos. Revista Ciencia y Trabajo, 0(48), 152-156.

Zárate, R. A. \& Matviuk, S. (2012). Inteligencia emocional y prácticas de liderazgo en las organizaciones colombianas. Cuadernos de Administración, 28(47), 89-102. 